\title{
AN EFFICIENT ROUTING APPROACH FOR AGGREGATED DATA TRANSMISSION ALONG WITH PERFORMANCE IMPROVEMENT IN WIRELESS SENSOR NETWORKS
}

\author{
Subhashini $\mathbf{S}^{1}$, Parani T $\mathbf{K}^{2}$ \\ ${ }^{1}$ Student, Electronics and Communication Department, Dhanalakshmi Srinivasan College of Engineering, TamilNadu, \\ India \\ ${ }^{2}$ Assistant Professor, Electronics and Communication Department, Dhanalakshmi Srinivasan College of Engineering, \\ TamilNadu, India
}

\begin{abstract}
Wireless Sensor Networks consist of large number of sensors which are deployed close to the proximity which is to be monitored. Sensors are energy-constrained devices, so it is very important to design a routing protocol for transmitting the aggregated data to the sink node effectively. In this paper, a novel approach called EDRINA (Enhanced Data Routing for In-Network Aggregation) is proposed. EDRINA forwards data using spanning tree in the cluster, where cluster head is elected using forward aware technique, towards the destination node. It has features like maximized data aggregation rate, low cost, low overhead, thus improves the network lifetime by reducing energy consumption. EDRINA is compared with Data Routing for In-Network Aggregation (DRINA), Shortest Path Tree (SPT) and Information Fusion based Role Assignment (InFRA) algorithms using Network Simulator and is proved to be outperforming in all the evaluated scenarios.
\end{abstract}

Keywords: Data Aggregation, Routing Protocol, Wireless Sensor Network

\section{INTRODUCTION}

Wireless Sensor Networks (WSN) consist of large number of sensors which are deployed in the environment to monitor various phenomenon's like pressure, temperature, sound, motion, or vibration in different locations. WSNs are widely used in many applications like monitoring critical infrastructure, home land security, environmental monitoring, communication, military and in many other critical applications[2].Sensor nodes in the network are energy-limited as they are battery operated and the energy consumption is generally associated with the data gathering and all other operations performed by the nodes. Among all the activities performed in the network, communication is the most expensive activity in terms of energy consumption [1].

Data-driven networks generate large amount of data [1], which are to be transmitted efficiently to the sink node. Transmission of large amount of data consumes more energy which decreases the network lifetime. In order to reduce the energy consumption, the data aggregation technique is performed, which in turn increases the network lifetime [6]. For routing the aggregated data, an efficient routing approach is required as the lose of aggregate data packet cause an immense impact on data inference at the monitoring center.
A novel approach called EDRINA (Enhanced Data Routing For In-Network Aggregation) is proposed here. It is a cluster based approach where the cluster head acts as the aggregation point and they transmit the packets only in the forward manner in the network. Spanning tree is formed in the cluster for forwarding the data. Thus it maximizes the data aggregation rate, reduces the tree cost, and reduces the overhead. This results in reducing the energy consumption for the communication cause the increased network lifetime.

\section{RELATED WORKS}

Various algorithms have been proposed to provide data aggregation during the routing in WSNs. Some of them are tree-based algorithms, cluster-based algorithms while others are simply structure-less algorithms [2].

\subsection{Shortest Path Tree (SPT) Algorithm}

It is a tree-based routing approach. It usually depends on hierarchical organization of the nodes. It uses a very simple strategy to construct the tree structure. Each source sends its information to the sink along the shortest path between the two [4]. Where these paths overlap for different sources, they are combined to form the aggregation tree. It has static routes [1]. 


\subsection{Information Fusion based Role Assignment}

\section{(InFRA) Algorithm}

It builds a cluster for each event including only those nodes that were able to detect it. Then, cluster-heads merge the data within the cluster and send the result toward the sink node[3]. The InFRA algorithm aims at building the shortest path tree that maximizes information fusion. Thus, once clusters are formed, cluster-heads choose the shortest path to the sink node that also maximizes information fusion by using the aggregated coordinators distance [3]. Main drawbacks of InFRA algorithm are very high overhead rate , low scalability and very high cost[1].

\subsection{Low Energy Adaptive Clustering Hierarchy (LEACH) Protocol.}

LEACH protocol is one of the most famous WSN hierarchical routing algorithms. In $\mathrm{LEACH}$, the nodes organize themselves into local cluster, the protocol is divided into a setup phase when the clusters are organized and a steady-state phase when data are transferred from the nodes to the cluster head and on to the sink [8]. LEACH-based algorithms assume that the sink can be reached by any node in only one hop, which limits the size of the network for which such protocols used [5].

\subsection{Data Routing For In-Network Aggregation (DRINA) Approach}

It is a cluster-based approach. The Cluster head $(\mathrm{CH})$ acts as the aggregation point. It aims at maximizing the overlapping routes for data transfer and reducing the communication cost. The drawbacks of DRINA are: the routes are static and the overhead is high [1].

\section{EDRINA: ENHANCED DATA ROUTING FOR IN-NETWORK AGGREGATION}

EDRINA is a cluster based approach, which mainly aims at improving the performance in the network while maximizing the data aggregation in the network. Here, $\mathrm{CH}$ acts as the aggregation point. It makes use of Periodic Simple Aggregation as its timing strategy for data aggregation. It requires each node to wait for a predefined period of time while aggregating all received data packet and, then, forward a single packet with the result of the aggregation [2].

For routing the data to the sink node from the source nodes clusters of sensors are formed in the network. In each cluster a node is elected as cluster head $(\mathrm{CH})$, which acts as the aggregation point. It receives data from all other nodes in the cluster for aggregation and forwards it to the destination via intermediate nodes which also includes $\mathrm{CH}$ of other clusters. Here, $\mathrm{CH}$ is elected on the basis of distance from the sink node i.e., the node closer to the sink will be elected.
Nodes can vary transmission power according to the distance to its receiver[8]. Initially the sink node broadcast to all the nodes in the network. The message broadcasted by the sink node will have the ID field containing the identifier of the node, DIST field which has the distance in hops from the sink node as shown in the Fig 1. Based on the DIST field value the node closer to the sink in each cluster can be obtained. Thus the $\mathrm{CH}$ will perform only forward data transmission towards the sink, which reduces the energy consumption in each node and improves the speed of transmission. This results in reduced delay in the aggregated data transmission.

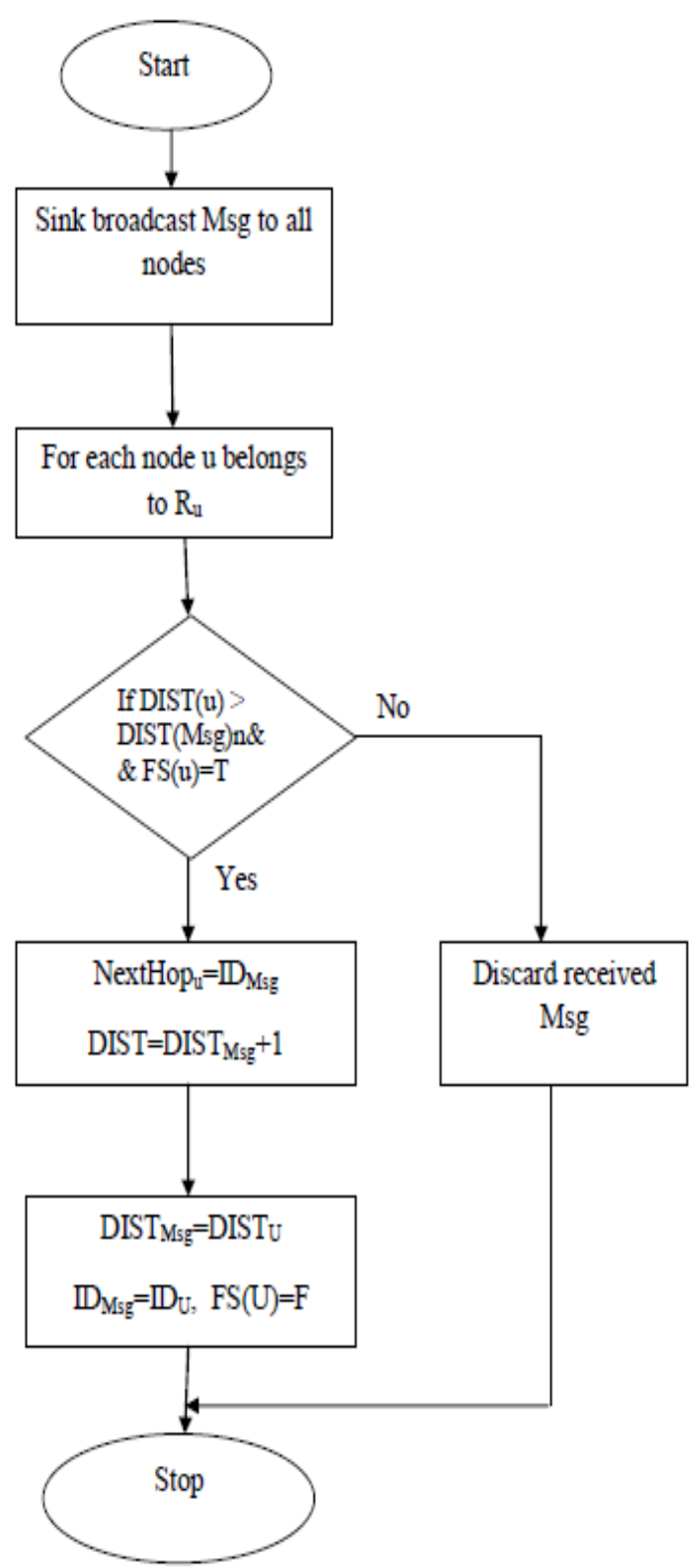

Fig 1 Flowchart for the initial message broadcasting for distance from sink estimation 
Spanning tree is constructed in the network for forwarding the data. The flow chart for the data transmission and tree formation is given in the Fig.2. In the tree, node with smallest identifier i.e., $\mathrm{CH}$ acts as root node. It exchanges the message containing the identifier of the node field, its root and the distance from the root in hops. The source node from which it receives the message is considered as the parent node. Identifier of the node is used to break the ties in the network. This algorithm makes use of shortest path for forwarding the data.

Timer is made use of for transmitting the message in a particular preset time. It helps for the maintenance of tree in the network. Whenever a message is transmitted, the timer P is started. It will get reset when there occurs any change in neighboring node. This approach helps to update the node itself and to transmit the message to the root node of the tree. Assume $r_{n}$ be the identifier of the root node for the node $n . d_{n}$ be the shortest distance from the node $n$ to $r_{n}, m_{n}$ be the message send by the node $n$ which consists of $n, r_{n}, d_{n}$ values, $p_{n}$ be the parent node ID for the node $n$ and $t_{\text {recv,n }}$ be the time node $n$ receive the message from the parent node as given in Fig 2. As the shortest path is used for data transmission, the energy usage is reduced and prolongs the network lifetime. The spanning tree used in the routing structure in cluster helps to improve the performance of the network. Performance of the proposed approach is compared with other known solutions in the following section

\section{PERFORMANCE EVALUATION}

EDRINA algorithm is evaluated and its performance is compared with other three known solutions: DRINA, SPT and InFRA algorithms. Performance metrics such as Efficiency, Overhead, Treecost are obtained by simulating the algorithm using Network Simulator version 2.34 , by varying the number of nodes in the network as well as the number of events sensed by the nodes. Following are the various parameters evaluated for the algorithms:

Data Packet Delivery Rate: This metric defines the quality of tree built by the algorithm. It denotes the number of packets reach the sink node. The lower the rate, higher will be the aggregation rate.

Control Packet Overhead: It denotes the number of control message used to set up the routing tree along with the cluster formation.

Efficiency: Packets per processed data. It denotes the rate between the number of packets transmitted to the number of packets received by the sink.

Routing Tree Cost:It denotes the number of edges in the routing tree structure built by the algorithm gives the treecost.

\subsection{Simulation Parameters}

Table 1. Simulation Parameters

\begin{tabular}{|l|l|}
\hline Simulation Parameter & \multicolumn{1}{|c|}{ Value } \\
\hline Simulator & NS v2.34 \\
\hline Topology Size & $1200 \mathrm{~m} \mathrm{x} \mathrm{1200m}$ \\
\hline Number of Nodes & 500 \\
\hline Communication Radius & $80 \mathrm{~m}$ \\
\hline \# of Events & 5 \\
\hline Simulation Duration & $4 \mathrm{hrs}$ \\
\hline Event Duration & $4 \mathrm{hrs}$ \\
\hline
\end{tabular}

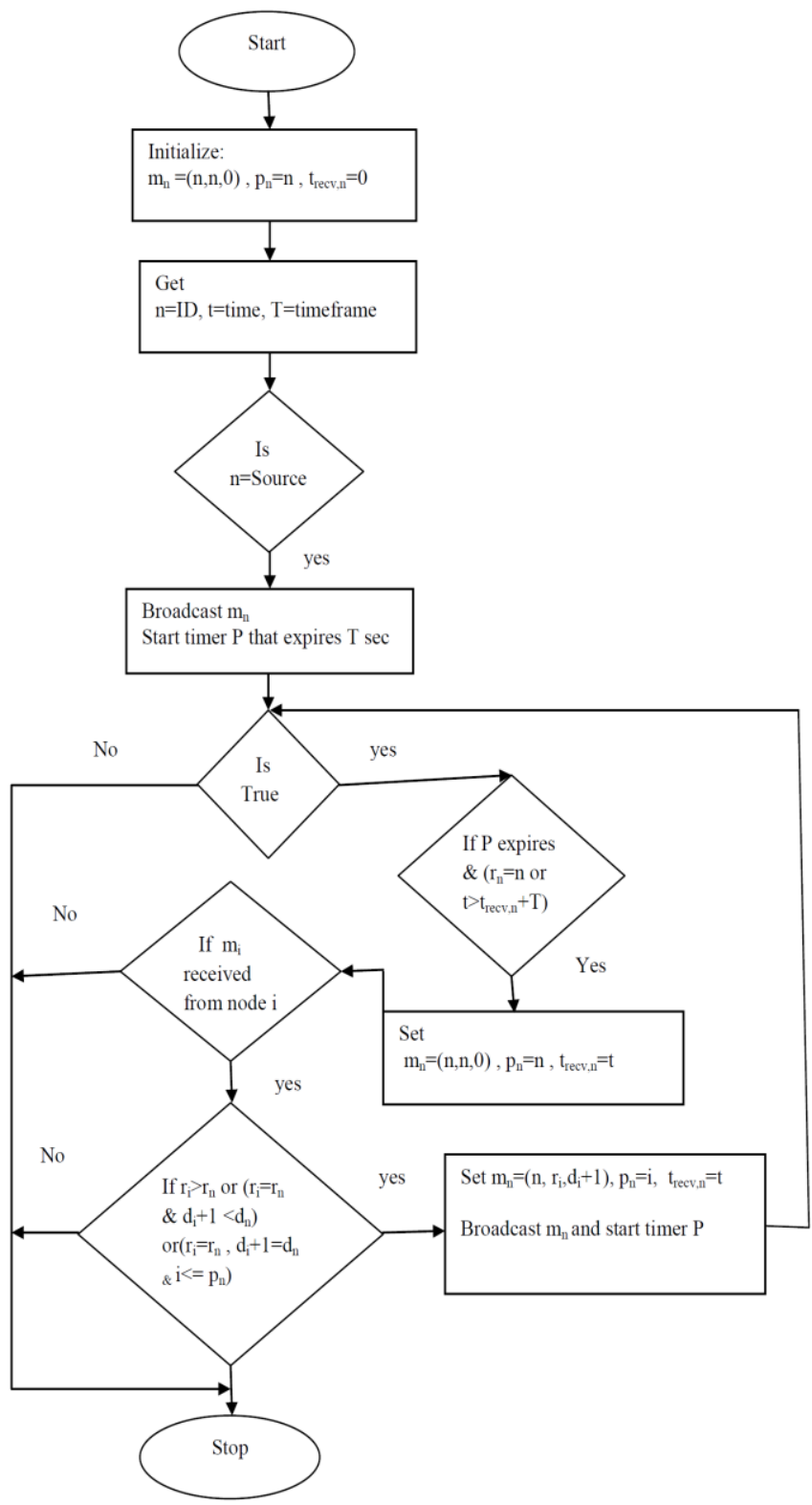

Fig 2. Flowchart for building and data transmission in the spanning tree. 


\section{RESULTS}

Each of the discussed performance metrics of the EDRINA algorithm are evaluated under various situations in the network for determining its efficiency. The algorithms performance is compared with the known protocols DRINA, SPT and InFRA under various criteria to out show its capability in various scenarios. For evaluating the performance of EDRINA in different scenarios the number of nodes in the network is varied from 100 to 500. Similarly, the number of events are varied from 1 to 5 for simulating the algorithm. The nodes sense all the events simultaneously in the network.

\subsection{Impact of Network Size}

For determining the impact of network size on the algorithms, the numbers of nodes are varied in the network. This helps to evaluate the algorithms scalability.

Fig 3 shows the efficiency of the EDRINA algorithm compared with other 3 known algorithms :DRINA, SPT and InFRA. Its clear that the number of packets per processed data is less for EDRINA compared to other algorithms proves that its efficiency is higher than that of DRINA and others.As the data aggregation rate of EDRINA is higher, the delay associated with the data transmission will get reduced. So, the aggregated data packets are transmitted with very less delay in the network deployed with EDRINA as routing approach than DRINA algorithm.

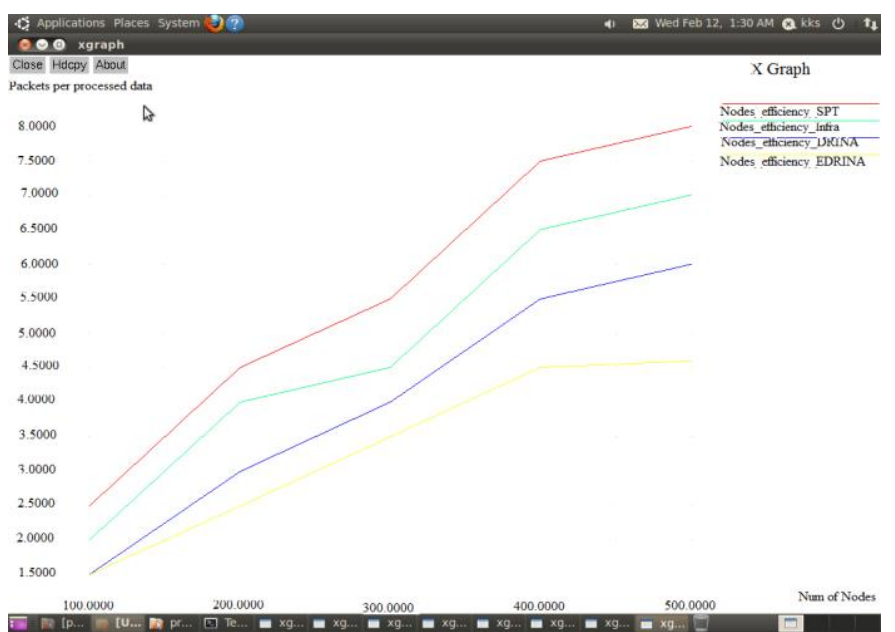

Fig 3 Efficiency: Packets per processed data vs number of nodes.

The graph shown in the Fig 4 gives the treecost of the algorithms. Number of edges required for EDRINA is very less when compared to SPT Whereas for DRINA it is medium value. Thus the routing tree for the EDRINA algorithm is proved as very efficient.

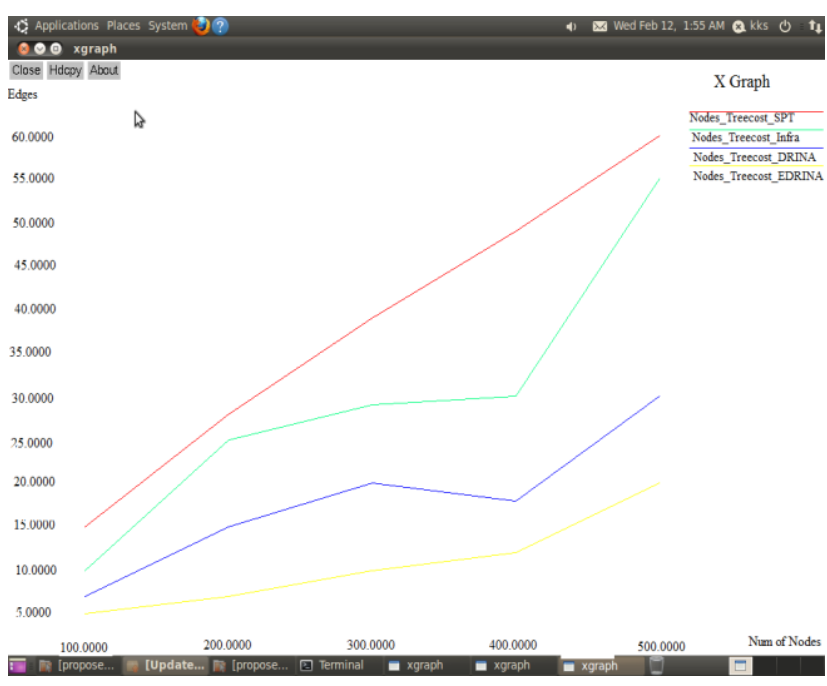

Fig 4 Treecost: Edges Vs Number of Nodes

Fig 5 shows the overhead for the EDRINA algorithm compared with other known solutions by varying the number of nodes in the network. It is a known fact that Overhead for DRINA is very large compared to others[1], whereas the overhead for the EDRINA is less, thus shows it requires less control message to built the routing structure than other algorithms. Thus it is proved that, EDRINA's performance is efficient even when the number of nodes are varied in the network.

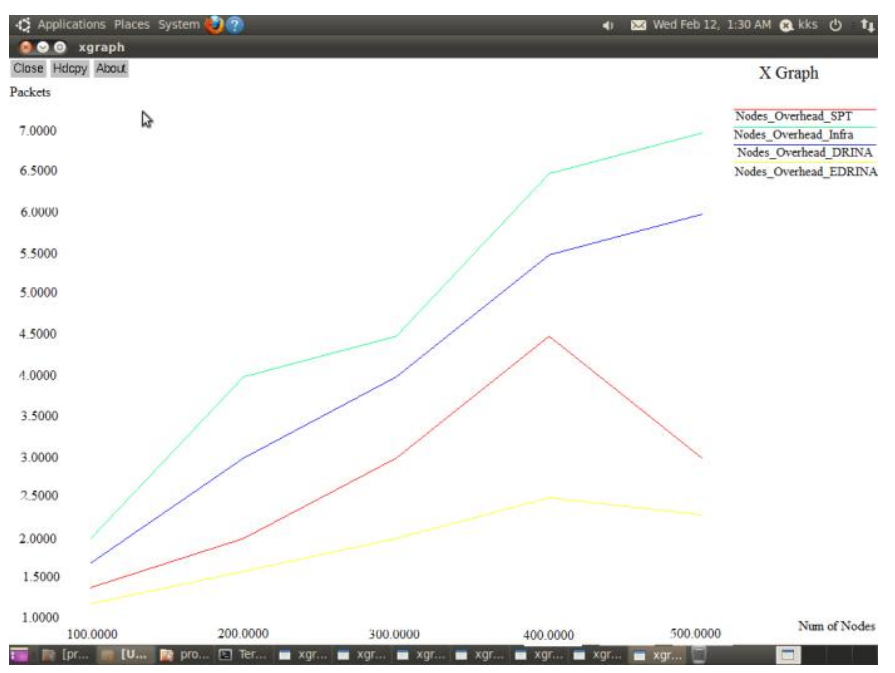

Fig 5 Overhead: Packets vs Number of Nodes

\subsection{Impact of Number of Events}

The performances of the algorithms are evaluated by varying the number of events sensed by the nodes in the network by keeping the number of nodes as constant. Numbers of events are varied from 1 to 5 for evaluation. 


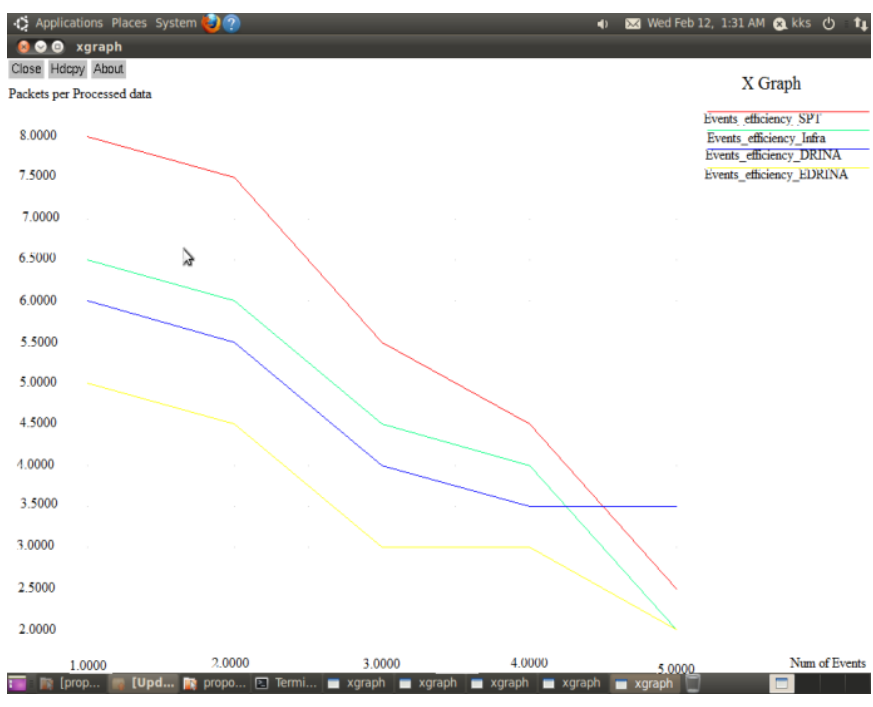

Fig 6 Efficiency: Packets per processed data vs Number of events.

The Fig 6 shows the graph indicating the efficiency of the EDRINA algorithm. The number of packets transmitted for the EDRINA is very less compared to that of SPT. The DRINA has less than that of Infra and SPT. Even when the events are increased the number of aggregated data packet transmission is less compared to that of others. This proves that EDRINA has an efficient routing structure with high aggregation rate compared to others. Thus its proved that the efficiency of the EDRINA is much better than the other known solutions.

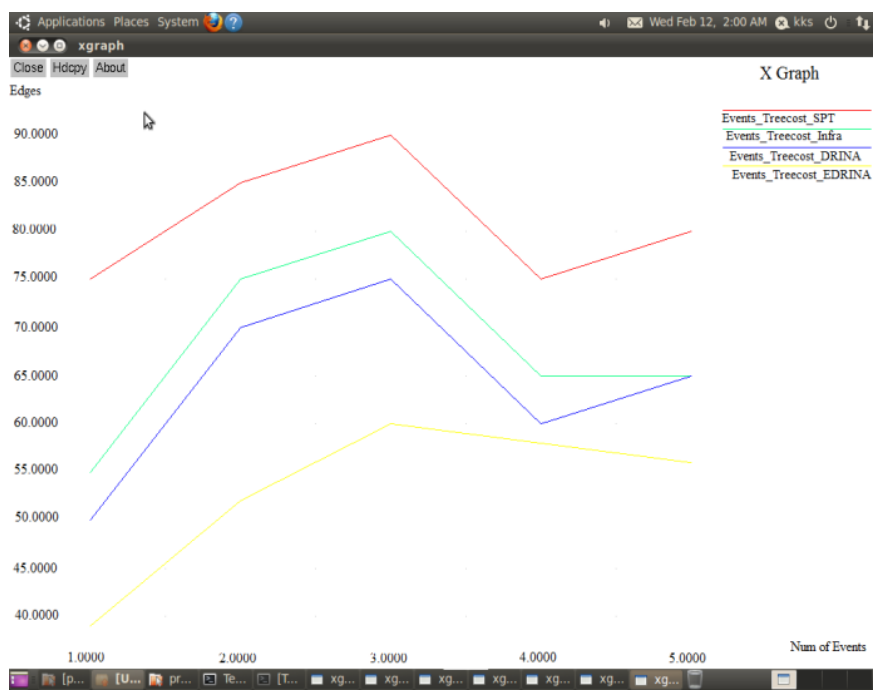

Fig 7 Treecost: Edges vs Number of Events

Treecost for the proposed algorithm is given in the Fig 7 . When the number of events are increased, the number of edges are increased in the structure. The number of edges required for the EDRINA is very less compared to that of DRINA, SPT and InFRA algorithms. When the number of edges required is less, the treecost for the algorithm will also get reduced. EDRINA has very lower treecost.

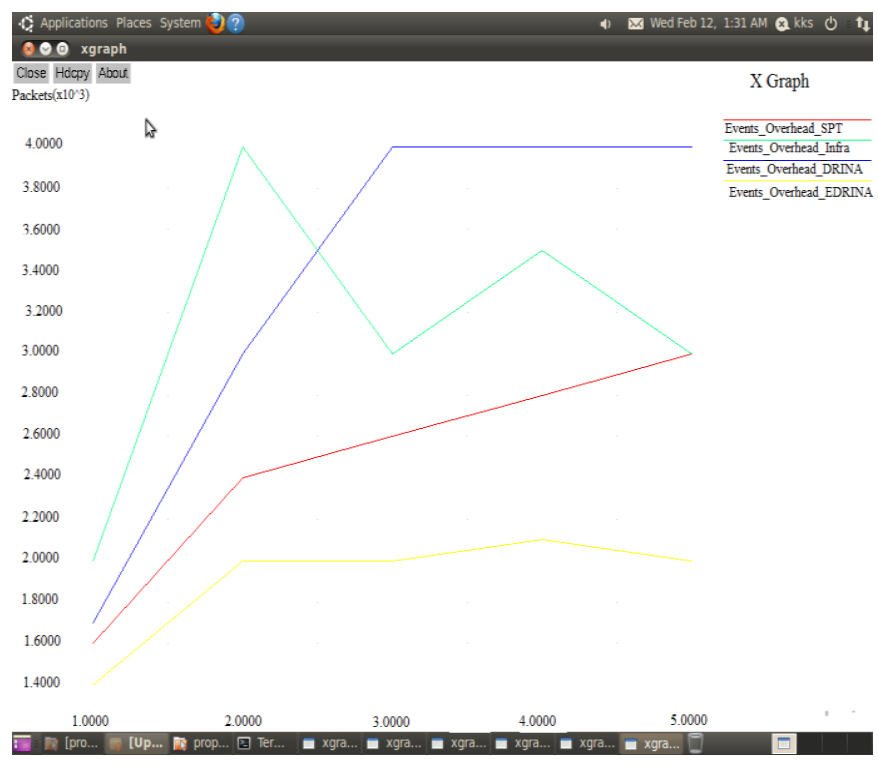

Fig 8 Overhead: Packets vs Number of Events

The graph in Fig 8 shows that the number of control packets required for the algorithm increases with increase in the number of events in the network. Proposed approach EDRINA requires less control message for the routing structure, as there occurs no flooding in the networks, the packets transmitted also very less. Thus, the packet overhead is very less for EDRINA and is maximum for DRINA. Decrease in the overhead improves the performance of the EDRINA algorithm and makes it very suitable for the routing of aggregated data packets in sensor networks.

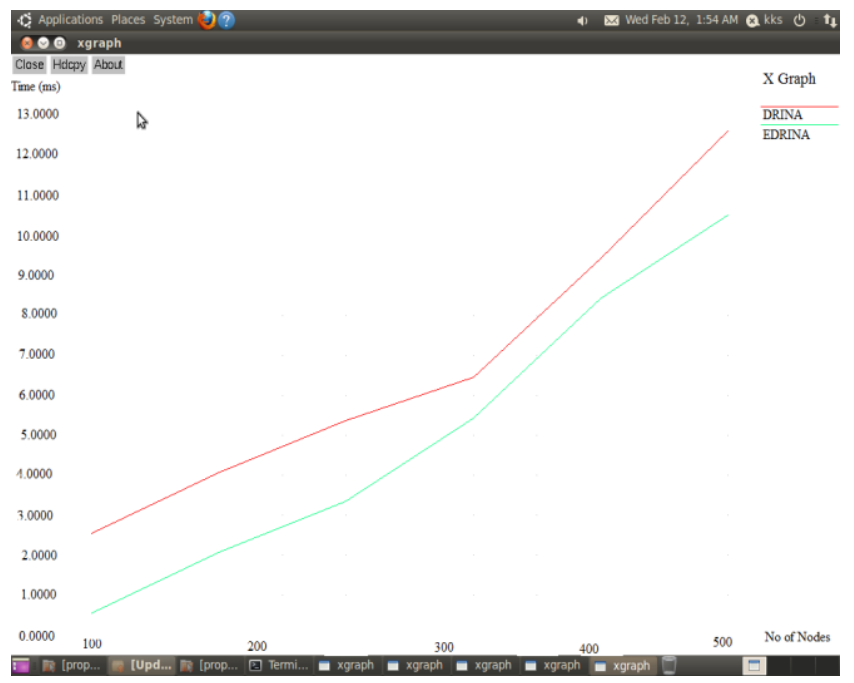

Fig 9 Delay: Time vs Number of Nodes 
The graph shown in the fig 9 denotes the delay associated with the data transmission in the network with DRINA and EDRINA approaches. From the graph its clear that the delay for EDRINA is less compared with the known solution. Thus it proves that the data transmission is faster in the network deployed with the proposed approach.

\section{CONCLUSIONS}

Wireless Sensor networks are widely used for monitoring various phenomenon in different applications. The proposed EDRINA algorithm is used for routing the aggregated data packets in WSNs. It is a cluster-based approach and makes use of spanning tree for routing the packets. It uses the technique of forward-aware routing for data transmission in the cluster. The EDRINA algorithm is compared with the other known solutions: DRINA, SPT and InFRA algorithms for the performance evaluation by varying the number of nodes and number of events sensed in the network. EDRINA approach significantly reduces the number of data transmissions so as to decrease the overhead. Simulation results shows that EDRINA outperforms the other known solutions in all the evaluated scenarios. Thus, its proved that EDRINA has higher aggregation rate, efficiency low overhead and delay. This decreases the energy consumption in the nodes lead to increased network lifetime. Its application can also be extended to the Mobile Wireless Sensor Networks. Data transmission has to be performed in a reliable way, EDRINA algorithm can be extended in such a way that it provides reliability in aggregated data transmission in the WSNs.

\section{REFERENCES}

[1] Villas L.A, Boukerche A, Ramos H.S, De and Loureiro A.A.F (2013)“DRINA: A Lightweight Aggregation in Wireless Sensor Networks," IEEETrans.,on computers, vol.62 No.4, pp 676-689.,2013

[2] K. Romer and F. Mattern, "The Design Space of Wireless Sensor Networks," IEEE Wireless Comm., vol. 11, no. 6, pp. 54-61, Dec. 2004.

[3] E.F. Nakamura, H.A.B.F. de Oliveira, L.F. Pontello, and A.A.F.Loureiro, "On Demand Role Assignment for Event-Detection inSensor Networks," Proc. IEEE 11th Symp. Computers and Comm. (ISCC '06), pp. 941947, 2006.

[4] B. Krishnamachari, D. Estrin, and S.B. Wicker, "The Impact of Data Aggregation in Wireless Sensor Networks," Proc. 22nd Int'l Conf. Distributed Computing Systems (ICDCSW '02), pp. 575-578,2002.

[5] A.P. Chandrakasan, A.C. Smith, and W.B. Heinzelman, "An Application-Specific Protocol Architecture for Wireless Microsensor Networks," IEEE Trans. Wireless Comm., vol. 1, no. 4, pp. 660-670, Oct. 2002.

[6] I. Chatzigiannakis, T. Dimitriou, S.E. Nikoletseas, and P.G. Spirakis, “A Probabilistic Algorithm for Efficient and Robust Data Propagation in Wireless Sensor
Networks," Ad Hoc Networks, vol. 4, no. 5, pp. 621635, 2006.

[7] Degan Zhang, Guang Li, ke Zhnag and Zhao-Hua Pan, "An Energy-Balanced Routing Method Based on Forward-Aware Factor in Wireless Sensor Networks" IEEE Trans. Industrial Informatics., Vol. 10, no. 1 pp. 766-773, Feb. 2104

[8] Marc Lee and Vincent W.S Wong, “ An Energy Aware Spanning Tree Algorithm For Data Aggregation in Wireless Sensor Networks," IEEE 2005.

[9] Anastasi G, Conti M, Francesco M, and Passarella A, "Energy Conservation in Wireless Sensor Networks: A Survey," Ad HocNetworks, vol.7,no. 3, pp. 537$568,2009$.

[10] Younis O, Krunz M, and Ramasubramanina "Node Clustering in Wireless Sensor Networks: Recent Developments and vol. 20, no. 3, pp. 20- 25,2006.

\section{BIOGRAPHIES}

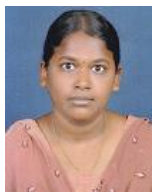

Subhashini $\mathrm{S}$ is currently persuing her Masters in Engineering from Dhanalakshmi Srinivasan College of Engineering, Coimbatore. She is interested in WSNs, MANET.

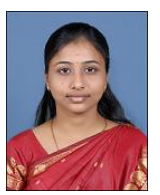

Parani $\mathrm{T} \mathrm{K}$ is currently working as an Assistant Professor in Dhanalakshmi Srinivasan College of Engineering, Coimbatore. Her area of interests are Wireless Communication and WSNs. 